\title{
The Effect of Active versus Passive Exploration on Memory for Spatial Location in Children
}

\author{
Audrey Feldman \\ State University of New York at Buffalo
}

\author{
Linda Acredolo
}

University of California, Davis

\begin{abstract}
Feldman, Audrey, and Acredolo, Linda. The Effect of Active versus Passive Exploration on Memory for Spatial Location in Children. Chind Development, 1979, 50, 698-704. The effect of active exploration upon memory for spatial location of an event was assessed for children at 2 age levels. Each child took a walk through the same unfamiliar hallway in search of a hidden object which he was later asked to relocate. Half the children were accompanied by an adult holding their hand (passive condition), while the other half proceeded on their own with an adult following behind (active condition). An age $\times$ condition interaction revealed that active exploration significantly improved performance of the 3- and 4-year-old group while not affecting the performance of the 9-and 10-year-olds. The 3- and 4-year-olds in the active condition, however, were still significantly less accurate than the older children, despite their experience of self-directed exploration. The results are interpreted as supporting the hypothesis that selfdirected activity serves to increase attention of preoperational children to relevant topological cues in the environment, whereas concrete operational children, due to their knowledge of projective and Euclidean space, demonstrate increased capacity to efficiently encode spatial information regardless of the mode of exploration.
\end{abstract}

Based on theoretical grounds (Gibson 1950), animal studies (Held \& Hein 1963), and anecdotal evidence, it has long been assumed that active exploration of an environment is likely to result in greater knowledge than passive exploration. Griffin (1973) contends, for example, "It is common experience that in a strange city, we remain much better oriented if we find our own way about than if we cover the same ground in the company of a local inhabitant who leads the way" ( $p$. 299). Since, according to Piagetian theory, it is primarily through active involvement with stimuli that a child's knowledge advances through the stages of cognitive development, this activepassive dimension assumes particular importance for developmental psychologists interested in how children gather information about the spatial layouts of environments. Yet this is one variable that has not yet been systematically examined in the context of the development of cognitive-mapping skills, even in those recent studies in which observations of children behaving in real-life spaces have replaced map drawing and paper and pencil spatial measures.
Indeed, it would seem reasonable to assume that such behavioral measures would be particularly influenced by the mode of exploration. As it stands, however, much of what little information we have from such studies stems from procedures in which the child has been limited to passive movement through the experimental space (e.g., Acredolo 1976; Acredolo, Pick, Olsen 1975). In the Acredolo et al. study, for example, subjects were required to remember the location at which an object had been dropped and retrieved during a guided stroll. The results indicated that the ability to recall the location improved with age and that the presence of a landmark near the target position significantly facilitated accuracy for preschoolers but not for 8-year-olds. In addition, it was found that accuracy at both ages was better when the children were forewarned that they would be asked to relocate the target position. While studies such as this one have revealed the existence of interesting age changes, the question remains whether these results are generalizable to situations in which the child is an active explorer of the environment. Per-

Portions of this article were presented at the biennial meeting of the Society for Research in Child Development, New Orleans, March 1977. Requests for reprints should be sent to Linda P. Acredolo, Department of Psychology, University of California, Davis, California 95616.

[Child Development, 1979, 50,698-704. (C) 1979 by the Society for Research in Child Development, Inc. 0009-3920/79/5003-0010\$00.75] 
haps, given the freedom of self-directed exploration and the increased attention to environmental cues it produces, the preschool child would garner as much information about an environment as the older child has been found to.

There is also the possibility that the mode of exploration will have a greater impact on the preoperational child as compared to the concrete operational child. According to Piaget, the preoperational child is restricted to topological notions of space, a system in which the presence of landmarks (for the noting of proximity relations) becomes particularly important. Consequently, any situation which reduces attention to these landmarks, passive exploration in this case, should result in relatively poor knowledge of the space. The concrete operational child, by contrast, also has the more sophisticated projective and Euclidean geometries to help familiarize a new space. According to Piaget, the advantage of Euclidean over topological knowledge is that the Euclidean concepts facilitate the establishment of "permanent relations spanning over greater spatiotemporal intervals, not merely within each successive perceptual field, but between each of these fields in turn" (Piaget \& Inhelder 1967, p. 418). In other words, the concrete operational child is better able to formulate a coordinated cognitive map in which relationships, not simply proximities to landmarks, play the central role. The concrete operational child is therefore in a much better position to draw inferences about the spatial layout from what information has been attended to. For example, a notion about the time a trip took or the number of corners turned and their angles or the directness of the route would provide valuable data to the older child. Finally, the older child would be more likely to be able to make a judgment about the layout of an environment based upon what layout would most likely be encountered in a given situation (e.g., most rooms have four walls, most buildings have four sides, most corners are $90^{\circ}$ angles, many trips start and stop in the same place). Thus, attention to large numbers of landmarks is simply not as crucial. Consequently, it may be that concrete operational children with their Euclidean and projective approaches to spatial problems may still be able to pick up enough information about a space during passive travel to form a cognitive map adequate to the task demands and significantly superior to that of the preoperational child.
The purpose of this study was to test these hypotheses concerning the effect of self-directed (active) versus other-directed (passive) exploration on the ability of children at two ages to master an unfamiliar spatial environment. The design, similar to that used by Acredolo et al. $(1975)$, required the subjects to try to remember the location where they had found an object during a walk through the space. Since we were mainly interested in the propensity for spontaneous retention of spatial information, the children were not warned ahead of time that this mernory task would be required. It was hypothesized that performance of the children allowed to move through the environment on their own (active group) would be better than that of the children who covered the same ground in the company of a guiding adult (passive group) and that this effect would be greater in the case of the 3-and 4-year-olds than for the 9- and 10-year-olds. In addition, for half the subjects in each group the site to be remembered was marked by a landmark. It was hypothesized that the presence of the landmark would prove particularly helpful in improving the accuracy of the younger children in the passive condition since it would provide an anchor upon which topological relations could be based even under conditions of reduced attention to the details of the total space.

A second purpose of the study was to look for characteristics which would differentiate between the accurate and inaccurate subjects within each group. Do the children who most readily generate accurate cognitive maps differ on other spatial or nonspatial dimensions from their less accurate age-mates? To discover if they do, the subjects were given a map-identification task, a question about the spatial layout of their home, a conservation-of-length task, and the Peabody Picture Vocabulary Test (PPVT). The conservation-of-length task was chosen as an index of intellectual development from a Piagetian viewpoint to contrast with the PPVT, a measure of intelligence in the traditional mental-testing sense. The specific choice of the conservation-of-length task was based upon its spatial character, its ease of administration, and the fact that it could be depended upon to result in variable performances at the preschool age level. Information about the correlates of good cognitive-mapping skills is not available in the literature to date and yet is crucial if we are ever to generate a comprehensive picture and accurate explanation of the development of cognitive mapping. 


\section{Method}

Subjects.-The subjects were 403 - and 4-year-olds from middle-class nursery schools, ranging in age from 3.25 years to 5.25 years with a mean of 4.33 years, and 409 - and 10year-olds from middle-class schools, ranging in age from 8.75 years to 11.17 years with a mean of 10.17 years. There were 20 males and 20 females at each age level, and 20 children were tested in each of the four experimental conditions, divided so that equal numbers of each age and sex were represented.

Procedure.-Memory for the location of an event was assessed in four experimental conditions which differed from one another in the type of exploration the child was allowed (active vs. passive) and in whether or not a landmark was present at the location to be remembered. The same unfamiliar hallway (see fig. 1) banked by office doors was used in each condition.

In the two passive conditions the child accompanied an adult on a walk in search of a hidden object. Before the walk began, the child was shown a cup like that for which he would be looking and was instructed, "Somewhere in this hall there's a cup just like this

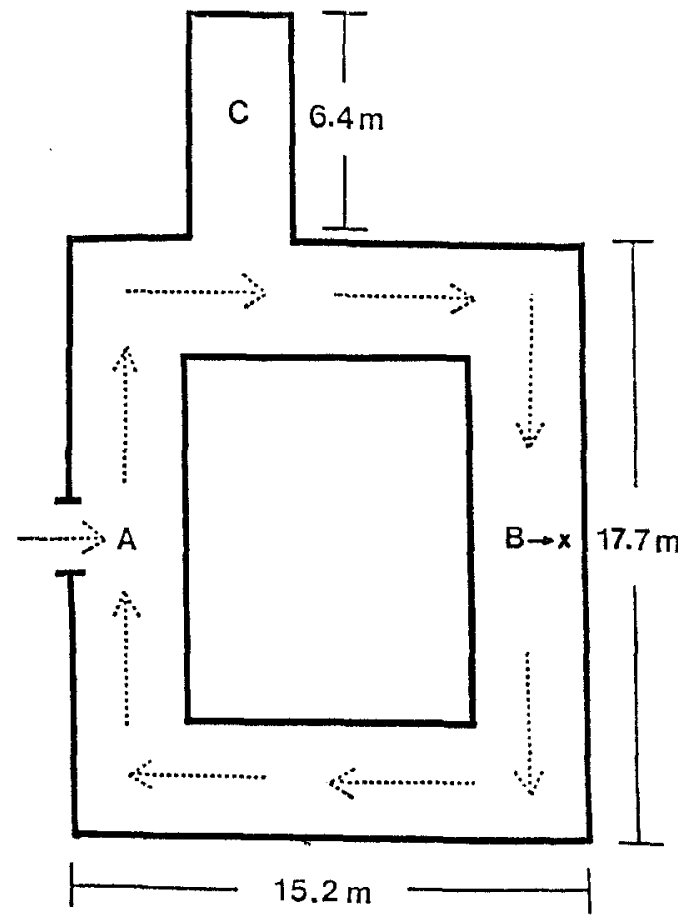

Fig. 1,-Experimental space one, and underneath it my office key is hidden. I'll take your hand and show you where to go. Your job will be to look for it." The adult held the child's right hand at all times so as not to block the object or other relevant cues on the child's left side from view. The walk began at point $A$ and continued until the child reached point $B$, where he found the hidden object. For half the children the location of the object was coded by a red square on the wall at the child's eye level. Once the child had found the object, he was prompted to continue around the hall to point $A$ (starting position) by the experimenter's suggestion that there might be another hidden object along the way. Upon reaching point $A$, at which time the child had had a full view of the experimental space, he was asked to return (by continuing in the same direction) to the exact place where the hidden object had been found.

As in the passive conditions, the children in the active conditions followed the path, beginning at point $A$, in search of a hidden object; however, in these conditions the children were instructed, "Somewhere in this hall my keys are hidden under a cup just like this one. You have to find your way around the hall yourself. I'll just be following behind." The children proceeded on their own with the adult following approximately the length of one hallway behind. If a child attempted to reverse direction at any time, he was encouraged to continue forward by the assurance that the object had not been missed. Once the child had found the object hidden at point $B$, he was prompted to continue to point $A$, as in the passive conditions, in search of any other hidden objects. Upon reaching $A$ the child was asked to continue (still unaccompanied and in the same direction) to the point where the object had been found. Again, for half the children the location was coded with a landmark.

Point $C$ on figure 1 represents a blind alley which the children in the active conditions had the option of entering. In order to control for the effect this experience might have on memory for point $B$, an equal number of children in the passive conditions were led into the alley. The decision as to whether or not to lead a child into the blind alley was determined by the choice made by the child immediately preceding him in the active conditions.

The time it took each child in the active conditions to find the object at point $B$ was recorded by stopwatch. For the children in the passive conditions the time to reach point $B$ 
was standardized: $45 \mathrm{sec}$ for the children who were led into path $C$ and $35 \mathrm{sec}$ for those who were not. Since the children in the active conditions did not have the opportunity to engage in conversation, an attempt was made to eliminate conversation in the passive conditions as well by asking the children to remain as silent as possible so as not to disturb the people at work in the offices. In actuality, the offices were empty.

For each child the distance between the actual location of the object and the location which the child chose was recorded. In addition, immediately following the walk in the hallway each child was asked to identify the best representation of the space from each of three sets of maps, each set varying a different dimension of the space. The five maps of set $A$ varied the basic configuration of the space (square, long rectangle, pentagon, triangle, or correct rectangle); the four maps of set $B$ varied the location of the hidden object; and the four maps of set $C$ varied the location of path $C$ (the blind alley). The PPVT and a conservation-of-length task were also administered to each child, as well as the following question designed to assess the child's knowledge of a familiar environment such as the home: "Pretend that you are in your bathroom at home brushing your teeth and looking in the bathroom mirror. If I change that mirror into a window so that you can see right through the bathroom wall, what room or part of your house would you be looking at?"

\section{Results}

The data consisted of the distance error in feet for each child. Presented in table 1 are the mean distance errors for each age group in each condition. As no significant sex differences were found at either age level, a 2 (age) $\times 2$ (landmark) $\times 2$ (condition) analysis of variance was performed (following a squareroot transformation), collapsing over sex. The analysis revealed significant main effects of age, the 9 - and 10-year-olds performing more accu- rately than the 3 - and 4-year-olds, $F(1,72)=$ $38.07, p<.01$, and condition, subjects in the active condition performing more accurately than those in the passive, $F(1,72)=9.58, p$ $<.01$. The interaction of age $\times$ condition was also significant, $F(1,72)=6.08, p<.05$, but the landmark $\times$ condition interaction was not, $F(1,72)=3.93, \quad p<.10$. A Newman-Keuls analysis of the age $\times$ condition interaction revealed that the active conditions had resulted in significantly greater accuracy than the passive conditions for the 3 - and 4-year-olds $(p<$ .01 ) but not for the 9- and 10-year-olds. However, despite the improved accuracy of the 3and 4-year-olds in the active conditions, these subjects were still significantly less accurate than the older subjects in both the active and passive conditions $(p$ 's $<.05)$. The $3-$ and 4 year-olds in the passive conditions were also significantly less accurate than both groups of 9 . and 10-year-olds ( $p$ 's $<.01$ ).

Although the length-conservation task and the map-identification task did differentiate the performances of the older and younger subjects $\left(v^{2}[1]=21.07, p<.005 ; x^{2}[1]=16.31, p<\right.$ .005 , respectively), neither measure was useful in differentiating the accurate responders (distance error below the mean of their group) from the inaccurate (distance error above the mean of their group) within a particular age level. Two measures which did differentiate successfully between the accurate and the inacurate 3- and 4-year-olds (but not the 9- and 10year-olds) were the PPVT and the home-environment question. Those preschool children who were categorized as accurate in placing the object had significantly higher scores on the Peabody $(t[34]=2.31, p<.025)$ and were more likely to respond correctly to the homeenvironment question (Fisher exact probability test, $p<.05)$. Unfortunately, these results for the home-environment question are based on only 20 of the 40 preschoolers. Parental verification was not available for the rest. These 20, however, represent a random sample of the subjects, and, consequently, the data are still included. The only sex difference found on any

TABLE 1

Mean Distance Errors for Both Age Groups in Each Condition

\begin{tabular}{|c|c|c|c|c|}
\hline \multirow[b]{2}{*}{ AGE } & \multicolumn{2}{|c|}{ Active } & \multicolumn{2}{|c|}{ Passtve } \\
\hline & Landmark & No Landmark & Landmark & No Landmark \\
\hline $\begin{array}{l}3-4 \text { уг } \ldots \ldots \ldots \ldots \\
9-10 \text { уг } \ldots \ldots \ldots\end{array}$ & $\begin{array}{r}10.5 \mathrm{ft}(3.2 \mathrm{~m}) \\
1.8 \mathrm{ft}(0.5 \mathrm{~m})\end{array}$ & $\begin{array}{l}7.4 \mathrm{ft}(2.3 \mathrm{~m}) \\
1.7 \mathrm{ft}(0.5 \mathrm{~m})\end{array}$ & $\begin{array}{r}17.9 \mathrm{ft}(5.5 \mathrm{~m}) \\
2.4 \mathrm{ft}(0.7 \mathrm{~m})\end{array}$ & $\begin{array}{c}40.7 \mathrm{ft}(12.4 \mathrm{~m}) \\
2.7 \mathrm{ft}(0.8 \mathrm{~m})\end{array}$ \\
\hline
\end{tabular}


measure was for map test $\mathbf{A}$ (basic configuration of the space), with 3- and 4-year-old males choosing the correct representation significantly more often than females (Fisher exact probability test, $p=.03$ ). In addition, the correlation between the time it took the subjects in the active conditions to reach point $B$ and accuracy in relocating point $B$ was not significant. Consequently, it appears that accuracy in relocating the target cannot be dismissed as merely a function of the length of time spent in the environment in search of the object.

\section{Discusgion}

An examination of the results for the spatial-location task indicates that, while the 9and 10-year-olds performed fairly consistently and very accurately across all conditions in the experiment, the 3- and 4-year-olds performed more accurately in the active conditions than in the passive conditions. Thus, the hypothesis that the younger children would benefit more than the older children from being allowed to actively explore the environment was confirmed. The results also indicated that the older children were consistently more accurate than the younger children in their memory for spatial location, even when the younger children were given the benefit of active exploration. The addition of a landmark did not significantly facilitate performance, although it clearly had its biggest impact in the hypothesized cell, namely, among the preschoolers in the passive conditions. This failure of the landmark to significantly affect memory contrasts with the results of Acredolo et al. (1975) and may be attributable to the specific landmark used in the present study, a geometrical form on the wall approximately 3 feet above the target object. In the Acredolo et al. study the landmarks used included a bright orange bench in the unfamiliar environment and a piece of jungle-gym equipment for the familiar environment. Both were located on the ground close to the target locations. It seems reasonable to assume that these latter objects would be more perceptually salient to the children than the one used in the present study and, therefore, more likely actually to function as landmarks. Finally, accuracy on the home-environment question and performance on the PPVT were found to be positively related for the 3-and 4-year-olds to accuracy in the spatial-memory task, while conservation of length was not.

Several alternative explanations for the differential effect of active exploration on the two age groups are possible. First, the results could be interpreted as supporting the hypothesis that for older children the level of attention to the external environment does not decrease during passive exploration, while for younger children it does. Perhaps, for example, younger children are more likely than older children to abrogate responsibility for the route to the guiding adult, thus turning their attention to nonspatial matters. After all, it is true that a preschooler spends a good deal of time attached (often involuntarily) to an adult on the way to and from places the child may have no wish to go. However, both common experience and empirical findings (Appleyard 1970; Griffin 1973) argue against such an explanation on the grounds that decreased attention under conditions of passive exploration is not restricted to young children but, rather, occurs across the age span.

As a second possibility there is the hypothesis that originally prompted the expectation of an age difference-namely, that the effect of the passive conditions was greater on the younger children because of their heavier dependence on the topological spatial informa. tion conveyed by environmental landmarks. In other words, the passive conditions may have resulted in decreased attention to the environment for both age groups, but the consequences of this decrease on memory for spatial location were more severe for the 3 - and 4-year-olds because it prohibited them from noting features of the environment upon which their topologically based memories could operate. Although the present results do not prove that this is the case, they are consistent with such an explanation.

This interpretation of the role of active movement is congruent with Olson's (1970) theory of the contribution of "performatory activities" to cognitive development. As a result of extensive experimentation with preschool children's ability to construct diagonal lines, Olson concluded that behaviors which involve the child in making decisions and choosing among alternatives are much more likely than other more passive experiences to result in the acquiring of perceptual information necessary to complete a task successfully. "Any performance is a sequential act. As such, it involves a continuous set of decisions at each point in time as to how to begin, how to continue, and how to terminate. Each of these decision points requires information. . . . It is performatory attempts ... that make it necessary for us to 
pick up certain other invariants from the perceptual world-invariant cues that would, and do, go undetected except for the attempts at mastery" (pp. 182, 188). In other words, it was an "attempt at mastery" which was provided the children in the active conditions of the present experiment when they were required to tour the space on their own looking for the object. But why did not this performatory experience, with its resulting increase in perceptual information, yield for the preschoolers performances in the subsequent memory task comparable to those of the 9- and 10-year-olds? Again one possibility, supported by Olson's research as well as Piaget's, is that the cues to which preschool children attend in a spatial task tend to be topological in nature rather than Euclidean. Furthermore, the topological information gathered in the course of the "performatory act" of initially touring the space was not necessarily the topological information best suited to relocating the object's position. The major advantage of Euclidean concepts in such a situation is that they enable children to organize isolated pieces of topological information into a coordinated system of interrelationships across time and space (Piaget \& Inhelder 1967). Such a coordinated overview of their experience in the space would increase the likelihood of accurate performance in the memory task. Thus, the fact that the 9- and 10-year-olds continued to outperform the preschoolers despite the younger children being given the opportunity to actively explore the space is not as surprising as it might first appear. Of course, the possibility remains that, had the environment been larger or the route more complex, a difference between the active and passive conditions might have resulted for the 9- and 10year-olds as well. However, given the present hypothesis regarding the contribution made by Euclidean concepts, we would predict that the degree to which the subjects would benefit from active exploration in such an environment would still be greater for preoperational than concrete operational children. Of course, verification of this prediction awaits further testing.

It is also important to consider the finding that the subsample of preschool children who demonstrated greater knowledge of their home environment also demonstrated more accurate knowledge of the unfamiliar environment. The importance of this finding is its consistency with the hypothesis that, rather than being an isolated, situation-specific performance, the children's accuracy on the spatial-memory task is quite likely a reflection of a more fundamental capacity to process information from large-scale spatial environments. In order to answer the home-environment question correctly, the child either had to have formed a cognitive map representing the relations between rooms or, at least, had to have noticed the juxtaposition of the two spaces involved. In either case, the child is indicating that spatial layout is a relatively important focus on his attention. Such a focus would surely be an asset in the spatial memory task in the unfamiliar environment, quite likely leading to more accurate performance.

Although the relationship between the home-environment question and accuracy in the unfamiliar space can be explained on the basis of spatial common denominators, the observed relationship between accuracy in the unfamiliar space and verbal intelligence obviously cannot. The most reasonable explanation seems to us to be that both measures are tapping the proverbial "general" or " $g$ " factor in intelligence. Despite the current trend toward stressing their independence, it is not the case that verbal scores and spatial scores are totally uncorrelated. Cronbach (1960), for example, reports that the vocabulary measure and the spatial measure of the General Aptitude Test Battery correlate at $r=.40$ and that this same vocabulary measure correlates even higher ( $r=$ .49 ) with the spatial relations scale of the Differential Aptitude Test. He also points out that the verbal and performance IQs of the WISC correlate very highly (.77 to .81$)$.

In summary, the present data clearly indicate that active exploration of an environment does facilitate memory for spatial information in preschoolers. This finding is important both practically and empirically. From a practical point of view, it may be important for parents and teachers to know the benefits of active exploration to the child's knowledge of spatial layout. In view of these results, it seems ironic that the main reason for not allowing children to move on their own is to prevent them from getting lost. From an empirical point of view, it is important that researchers in this area be aware that the mode of exploring a space will affect the performance of young children. However, it is also important to point out that allowing active exploration did not eliminate the difference in accuracy between the older and younger children. Consequently, the results of the present study tend to confirm rather than 


\section{Child Development}

invalidate conclusions about age differences resulting from similar studies using passive exploration (Acredolo et al. 1975). In fact, the replication of their results in a different environmental setting is reassuring evidence of their validity.

\section{References}

Acredolo, L. P. Frames of reference used by children for orientation in an unfamiliar space. In G. Moore \& R. Golledge (Eds.), Environmental knowing. Stroudsburg, Pa.: Dowden, Hutchinson \& Ross, 1976.

Acredolo, L. P.; Pick, H. L.; \& Olsen, M. G. Environmental differentiation and familiarity as determinants of children's memory for spatial location. Developmental Psychology, 1975, 11, 495-501.
Appleyard, D. Styles and methods of structuring a city. Environment and Behavior, 1970, 2, 100118.

Cronbach, L. J. Essentials of psychological testing. New York: Harper \& Row, 1960.

Gibson, J. J. The perception of the visual world. Boston: Houghton Mifllin, 1950.

Griffin, D. R. Topographical orientation. In R. Downs \& D. Stea (Eds.), Image and environment: cognitive mapping and spatial behavior. Chicago: Aldine, 1973.

Held, R., \& Hein, A. Movement produced stimulation in the development of visually guided behavior. Journal of Comparative and Physiological Psychology, 1963, 56, 872-876.

Olson, D. R. Cognitive development. New York: Academic Press, 1970.

Piaget, J., \& Inhelder, B. The child's conception of space. New York: Norton, 1967. 
This document is a scanned copy of a printed document. No warranty is given about the accuracy of the copy. Users should refer to the original published version of the material. 